\title{
Projeto de Controle Ótimo Baseado em Observador de Distúrbio com Uso de Otimização Evolutiva *
}

\author{
Denise Fonseca Pereira* Eduardo Nunes Gonçalves** \\ * Programa de Pós-graduação em Engenharia Elétrica \\ UFSJ/CEFET-MG Avenida Amazonas 7675, Belo Horizonte, MG, \\ Brasil (e-mail: pereira.denisede@gmail.com). \\ ** Departamento de Engenharia Elétrica - CEFET-MG Avenida \\ Amazonas 7675, Belo Horizonte, MG, Brasil (e-mail: \\ eduardong@des.cefetmg.br)
}

\begin{abstract}
In this work, it is propose a design method of control based on disturbance observer in the frequency domain. The control problem is formulated as a multiobjective optimization problem in which the optimization variables are the parameters of the controller and the disturbance observer. In order to have a compromise between disturbance rejection and measurement noise attenuation, with consequent minimization of the control effort, we have adopted as control objectives the $\mathcal{H}_{\infty}$ norm of the transfer function relating the disturbance and the system output and $\mathcal{H}_{2}$ norm relating the measurement noise and the control signal. A multiobjective evolutionary optimization technique is applied to provide a set of efficient solutions with different trade-offs between the two control objectives, allowing the designer to determine which solution is most suitable for the specific problem.

Resumo: Neste trabalho é proposto um método de projeto de controle baseado em observador de distúrbio, no domínio da frequência. Formulou-se o problema de controle como um problema de otimização multiobjetivo em que as variáveis de otimização são os parâmetros do controlador e do observador de distúrbio. De modo a se ter um compromisso entre rejeição ao distúrbio e atenuação de ruído de medição, com consequente minimização do esforço de controle, nós adotamos como objetivos de controle a norma $\mathcal{H}_{\infty}$ da função de transferência relacionando o distúrbio e a saída do sistema e a norma $\mathcal{H}_{2}$ relacionando o ruído de medição e o sinal de controle. É aplicada uma técnica de otimização evolutiva multiobjetivo que fornece um conjunto de soluções eficientes com diferentes compromissos entre os dois objetivos de controle, permitindo ao projetista determinar qual é a solução mais adequada para o problema específico.
\end{abstract}

Keywords: Optimization in Control and Automation systems; control based on disturbance observer; Multiobjective evolutionary algorithm.

Palavras-chaves: Otimização em sistemas de controle e automação; Controle baseado em observador de distúrbio; Algoritmo evolucionário multiobjetivo.

\section{INTRODUÇÃO}

Um dos desafios existentes no controle de sistemas industriais está diretamente ligado ao controle dos distúrbios não medidos, isso devido ao fato da inviabilidade física ou financeira de instalação de sensores para obter tais dados. Esses processos de produção, sejam eles siderúrgicos, metalúrgicos, químicos, petroquímicos, dentre outros, constantemente estão expostos às interferências do ambiente externo. Essas interferências estão ligadas, por exemplo, às diferenças de temperatura entre o processo e ambiente, às características qualitativas dos insumos do processo, às vibrações de máquinas, e assim por diante. $\mathrm{O}$ que pode causar baixa qualidade do produto final. (Chen et al. (2016))

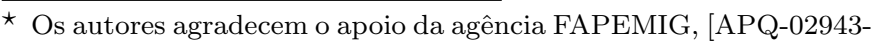
15] - Edital 01/2015 - Demanda Universal
}

O controle automático de sistemas é fundamental para o correto e adequado funcionamento de um processo, planta industrial ou equipamento, a fim de manter os valores das variáveis de saída dentro de limites operacionais predeterminados, assim a rejeição de distúrbios torna-se um ponto chave no projeto de controle devido aos efeitos causados nos sistemas.

As técnicas utilizadas nos observadores de distúrbio objetivam estimar a perturbação do ambiente externo.O observador de distúrbio, abreviado por DOB (do inglês, Disturbance Observer) foi inicialmente proposto por Ohishi et al. (1983) para o controle de velocidade de um motor DC. A princípio, a pesquisa objetivava compensar os efeitos que o torque de carga desconhecido exercia sobre o motor, porém, com o controle até então realizado, o sistema apresentava erros, tanto em estado estacionário quanto transitório. Implementando o método baseado na 
teoria de observadores foi possível estimar o somatório dos distúrbios existentes e desenvolver um controlador de torque que reduzisse o erro na resposta transitória e eliminar o erro em regime permanente.

Desde a década de 80, muitos estudos têm sido desenvolvidos a fim de explorar as características ainda não estudadas e aprimorar os algoritmos dos observadores de distúrbio. Em Chen (2004), um método de controle baseado em observador de perturbação foi implementado para o projeto de observador de perturbações não lineares aplicado a uma classe de sistemas não lineares de única entrada e única saída (SISO, do inglês, single-input and singleoutput). Já em Guo and Chen (2005), novas leis de controle baseadas no observador de perturbação são propostas para estimar e rejeitar perturbações de sistemas não-lineares de múltiplas entradas e múltiplas saídas (MIMO, do inglês, multiple-input and multiple-output).

Recentemente, An et al. (2016) utilizaram uma combinação do controle baseado em DOB com uma técnica de linearização por realimentação para regular a velocidade de veículos hipersônicos sujeitos à distúrbios na entrada. Da mesma forma, Mi et al. (2016) projetaram um controlador de frequência de carga de modo deslizante para um sistema de energia híbrido incerto utilizando um observador de distúrbio para melhorar a precisão do controlador de frequência de carga e compensar as vibrações.Wang and $\mathrm{Su}$ (2015) desenvolveu uma pesquisa cuja finalidade é realizar o controle de sistemas estáveis de fase não mínimas e com atraso utilizando a estrutura DOB. Seus parâmetros do controlador e constante do filtro foram determinados utilizando procedimentos de otimização por padrão $\mathcal{H}_{\infty}$.

A contribuição deste trabalho é aplicar um método de otimização multiobjetivo para projetar simultaneamente os parâmetros do controlador PI e a constante do filtro passa baixa do observador de perturbação. O objetivo de controle é estabelecer um compromisso entre a rejeição à perturbação e a minimização do esforço de controle e, consequentemente, atenuação do ruído. Através desse método proposto será gerado um conjunto de soluções com diferentes compromissos entre esses dois objetivos dando ao projetista o critério de escolha das melhores condições do projeto e recursos disponíveis para implementação.

Este trabalho está organizado como descrito a seguir. A seção 2 apresenta uma breve abordagem sobre as características do DOB. Na seção 3 são apresentadas as várias funções de transferência em malha-fechada de interesse e é formulado o problema de síntese como um problema de otimização multiobjetivo. A seção 4 apresenta o método de otimização multiobjetivo a ser utilizado para a solução do problema formulado. Na seção 5 , o método de síntese proposto é avaliado por meio de um exemplo ilustrativo. A conclusão é apresentada na seção 6 deste trabalho.

\section{OBSERVADOR DE DISTÚRBIO}

O DOB tem por finalidade estabelecer, de uma forma eficaz e sem sensores, uma estimativa das perturbações presentes em uma planta ou processo e melhorar a robustez do sistema em malha fechada. (Chen et al. (2016))

Considere o diagrama de blocos do DOB apresentado na figura 1, cuja planta a ser controlada é $G(s)$. O DOB

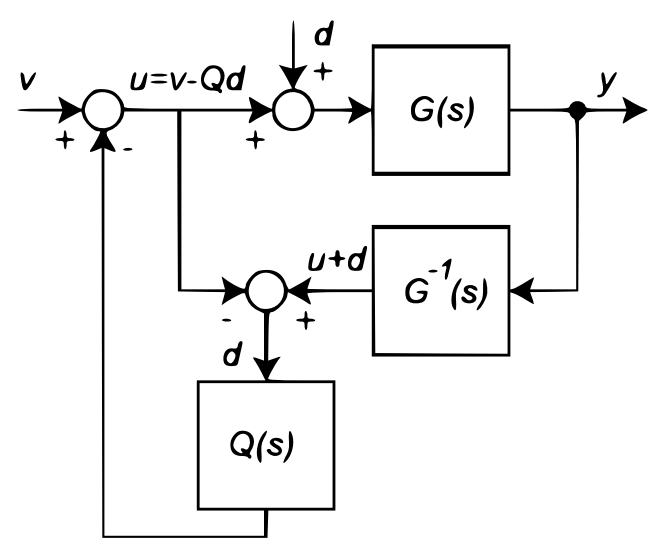

Figura 1. Diagrama de blocos do observador de distúrbio

é composto por um bloco correspondente ao inverso da planta, $G^{-1}(s)$, e um filtro passa-baixas, $Q(s)$. Devido ao uso da inversa da planta, essa configuração restringese à plantas de fase mínima. Desta forma, é possível compreender que o sinal de distúrbio inserido no sistema será cancelado ao passar pelo observador.

A partir da análise da figura 1, é possível extrair a função de transferência que relaciona o sinal de entrada $V(s)$, que é a saída do controlador, com a saída da plante $Y(s)$, considerando o filtro $Q(s)$ igual a um, como:

$$
\frac{Y(s)}{V(s)}=G(s)
$$

Assim, a inclusão do DOB no sistema não afetará a planta, pois a função de transferência do sistema original não é afetada. Ao realizar algumas manipulações no diagrama de bloco do DOB, apresentado na figura 1, têm-se uma configuração equivalente implementável, conforme representado pela figura 2.

O projeto do filtro determinará o desempenho do DOB em relação à rejeição de perturbação, assim este é escolhido como um filtro passa-baixa, devido ao fato de que a perturbação $d$ a ser estimada é de baixa ou média frequência. Se a planta a ser controlada estiver sujeita a perturbações de alta frequência, essa componente é, normalmente, filtrada pelo próprio sistema físico.

Este filtro deve ser projetado de forma que seu grau relativo não seja menor do que o grau relativo da planta, $G(s)$, de modo que o produto $Q(s) G_{n}^{-1}(s)$ seja uma função própria, isto é, o número de polos seja pelo menos igual ao número de zeros. Isso assegura que a estrutura seja realizável e seja possível implementar do inverso da planta.

O filtro $Q(s)$, a ser projetado, deve se aproximar de 1 , para que assim a perturbação estimada e a perturbação de entrada sejam aproximadamente iguais, cancelando seu efeito no sistema. Por outro lado, como a inversa da planta corresponde a um filtro passa-altas, para atenuar os efeitos dos ruídos de medição, o filtro $Q(s)$ deve ser projetado para atender ao compromisso entre rejeição de distúrbio e atenuação de ruídos. O filtro $Q(s)$ pode ser adotado como:

$$
G(s)=\frac{1}{\left(T_{q}+1\right)^{q}}
$$




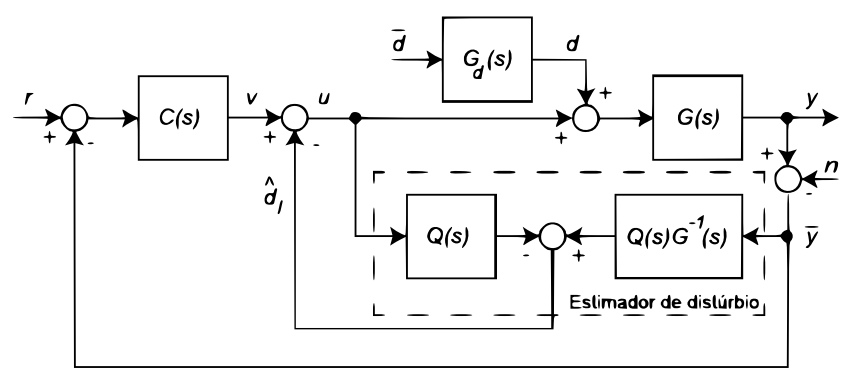

Figura 2. Diagrama de blocos do sistema de controle com o observador de distúrbios

Sendo $T_{q}$ a constante de tempo do filtro, a ser projetada, e $q$ a ordem relativa do filtro, a ser escolhida para assegurar que o produto $Q(s) G_{n}^{-1}(s)$ seja uma função própria.

\section{DESCRIÇÃO DO PROBLEMA}

O DOB é utilizado em uma aplicação no domínio da frequência a fim de estimar a perturbação presente no sistema, porém, o sinal de saída possui ruído em sua medição. Para tratar o sinal de ruído e estabelecer um compromisso entre a rejeição de distúrbio e atenuação de ruído de medição, o problema de controle será formulado como um problema de otimização multiobjetivo.

O sistema de controle baseado em DOB é expresso pelo diagrama de blocos da figura 2 , sendo $G(s)$ a representação da planta física real do sistema, $G_{n}^{-1}(s)$ é o inverso do modelo nominal da planta, $Q(s)$ e $C(s)$ são o filtro e o controlador, respectivamente, a serem projetados, $v$ é o sinal de saída do controlador, $u$ é o sinal de entrada de controle, $y$ é o sinal de saída do sistema, $r$ é o sinal de referência, $\bar{y}$ é a saída medida, $n$ é o ruído de medição, $d$ é o sinal de perturbação externa, $d_{l}$ é a perturbação combinada e $\widehat{d}_{l}$ é a estimativa da perturbação combinada.

Este sistema de controle possui dois graus de liberdade. $\mathrm{O}$ DOB tem a finalidade de estimar e rejeitar a perturbação e o controlador, por sua vez, deve ser ajustado para melhorar o desempenho de rastreamento. Porém, os objetivos de controle conflitam na escolha da resposta mais rápida possível para referência, de forma a minimizar os efeitos da perturbação e limitar o esforço de controle e os efeitos do ruído.

Considerando o diagrama de blocos da figura 2, é possível extrair as equações necessárias para a simulação e otimização dos parâmetros do sistema. O esforço de controle $u$ é dado por:

$$
u=\frac{C}{H} r-\frac{C G+G Q G_{n}^{-1}}{H} d+\frac{C+Q G_{n}^{-1}}{H} n
$$

A saída $y$ do sistema é calculada como:

$$
y=\frac{G C}{H} r+\frac{G(1-Q)}{H} d+\frac{G\left(C+Q G_{n}^{-1}\right)}{H} n
$$

Em que $\mathrm{H}$ é dado por:

$$
H=1+G C+G Q G_{n}^{-1}-Q
$$

Já o erro de rastreamento pode ser expresso por:

$$
e=r-y
$$

Substituindo (4) em (6), tem-se que o erro de rastreamento pode ser reescrito como:

$$
e=\frac{H-G C}{H} r-\frac{G(1-Q)}{H} d-\frac{G\left(C+Q G_{n}^{-1}\right)}{H} n
$$

Foram testadas diferentes escolhas de funções de transferência para serem consideradas na formulação do problema de controle. Para este problema, foi adotada a seguinte formulação, que apresentou os melhores resultados,

$$
x^{*}=\arg \min _{x}\left[\begin{array}{c}
\left\|T_{y d}(s, x)\right\|_{\infty} \\
\left\|W_{u}(s) T_{u n}(s, x)\right\|_{2}
\end{array}\right]
$$

Sujeito a:

$$
\begin{gathered}
x \in D \subset \Re^{n} \\
g=\operatorname{Real}\left(\operatorname{polos}\left(T_{y d}\right)\right)
\end{gathered}
$$

Sendo $D$ o hiper-retângulo que define a variação dos parâmetros do vetor $x$ :

$$
x=\left[K_{p} \frac{1}{T_{i}} T_{q}\right]^{T}
$$

A função de ponderação, $W_{u}(s)$, para um filtro passabaixas com frequência de corte elevada, é necessária para transformar o produto $W_{u}(s) T_{u n}(s)$ em uma função estritamente própria, como norma 2 finita

\section{OTIMIZAÇÃO EVOLUCIONÁRIA MULTIOBJETIVO}

Como o problema (8) envolve critérios conflitantes, o uso de técnicas de otimização multiobjetivo é aplicável a fim de obter os parâmetros ótimos do controlador e do filtro que serão usados, equação (10). Problemas que envolvem a otimização de duas ou mais funções objetivo conflitantes não possuem uma solução única. As técnicas que resolvem este tipo de problemas fornecerão um conjunto de soluções eficientes ou não dominadas ou conjunto Pareto.

A otimização multiobjetivo tem por finalidade encontrar o conjunto de soluções não dominadas ou eficientes, $\mathcal{X}^{*}$. Seja $\mathcal{F}_{x}$ o conjunto de soluções factíveis. Uma solução $x^{*} \in \mathcal{F}_{x}$ é eficiente se $f\left(x^{*}\right) \leq f(x)$ e $f\left(x^{*}\right) \neq f(x)$, isto é, não existe outra solução factível $x$ que seja melhor ou igual em todos os objetivos do que a solução $x^{*}$.

Esse conjunto de soluções eficientes pode ser gerado por meio de algoritmos de otimização multiobjetivo. Algoritmos de otimização evolutivos (EAs) podem ser adaptados para tratar problemas multiobjetivo, como por exemplo, o Algoritmo Genético de Ordenação Não Dominado (NSGAII, do inglês, Nondominated Sorting Genetic Algorithm), proposto por Deb et al. (2002), Algoritmo Evolutivo da Força de Pareto (SPEA2, do inglês, Strength Pareto Evolutionary Algorithm) por Zitzler et al. (2001) e o algoritmo Evolução Diferencial para Otimização Multiobjetivo 
(DEMO, do inglês, Differential Evolution for Multiobjective Optimization), proposto por Robič and Filipič (2005). Para resolver o problema multiobjetivo deste trabalho, foi aplicado o algoritmo DEMO.

O DEMO é fundamentado em um simples e eficiente algoritmo denominado algoritmo Evolucionário Diferencial (DE, do inglês, Differential Evolution) proposto por Price and Storn (1997), que é utilizado para resolver problemas de otimização de um único objetivo. No DE quando uma solução candidata, gerada por operadores de mutação e recombinação, é melhor, esta substitui a solução ancestral. Porém quando são vários objetivos, no DEMO, a solução candidata domina a ancestral, ela a substitui na nova geração. Se uma não dominar a outra, a solução candidata é incluída na população, aumentando a mesma. Quando isso acontece, é aplicado a método de seleção do NSGA-II para eliminar as piores soluções excedentes.

Assim como o DE, o DEMO possui etapas de mutação, cruzamento e seleção para criar uma nova geração, como pode ser visto em detalhes em Storn and Price (1997). A população inicial é criada, com $N$ indivíduos, de forma aleatória e distribuídos dentro do espaço inicial de soluções, considerando seus limites superiores e inferiores. Seja $x \in \Re^{n}$, sendo $n$ o número de variáveis de otimização, e definindo a $K$-ésima população como $X_{k}=\left(x_{k, i} ; i=1 \cdots N\right)$, em que:

$$
X_{k, i}=\left[\begin{array}{c}
X_{k, i, 1} \\
\vdots \\
X_{k, i, n}
\end{array}\right]
$$

$\mathrm{Na}$ etapa de mutação diferencial, sorteia-se três indivíduos da população $X_{k}, x_{r 1}, x_{r 2}$ e $x_{r 3}$, garantindo que $r 1 \neq r \mathscr{2} \neq r_{3} \neq i \in\{1, \cdots, N\}$, e é gerado um vetor mutante calculado como $v_{k, i}=x_{k, r 1}+F_{i}\left(x_{k, r 2}-x_{k, r 3}\right)$, sendo $F_{i} \in[0,5 ; 1]$ um fator de escala aleatório.

Na etapa de recombinação ou cruzamento para cada indivíduo da população é criado um vetor teste, $u_{k}$, por meio da recombinação do vetor ancestral, $x_{k}$, e o vetor mutante, $v_{k}$, que terá uma certa probabilidade de se recombinarem, para este trabalho a porcentagem utilizada foi de $90 \%$.

Como mencionado, a diferença do DE, para problemas com um objetivo, e o DEMO, é o operador de seleção, em que é considerado o conceito de dominância. Quando a população cresce além de $N$, é aplicado o ordenamento de fronteiras e o índice de aglomeração para reduzir a população novamente para $N$. (Deb et al. (2002))

\section{EXEMPLO ILUSTRATIVO}

Para exemplificar o uso do método proposta para projeto de controle com DOB, foi considerada uma parte do processo experimental apresentado por Johansson (2000). No trabalho desenvolvido por Johansson constituído por uma planta laboratorial com quatro tanques interconectados e duas bombas utilizadas para bombear o fluido para dentro dos tanques, formando uma estrutura como mostrado na figura 3. O objetivo é controlar o nível dos dois tanques inferiores a partir da tensão aplicada nas bombas. A variação da vazão da bomba em um dos lados afeta também o outro lado e vice-versa.

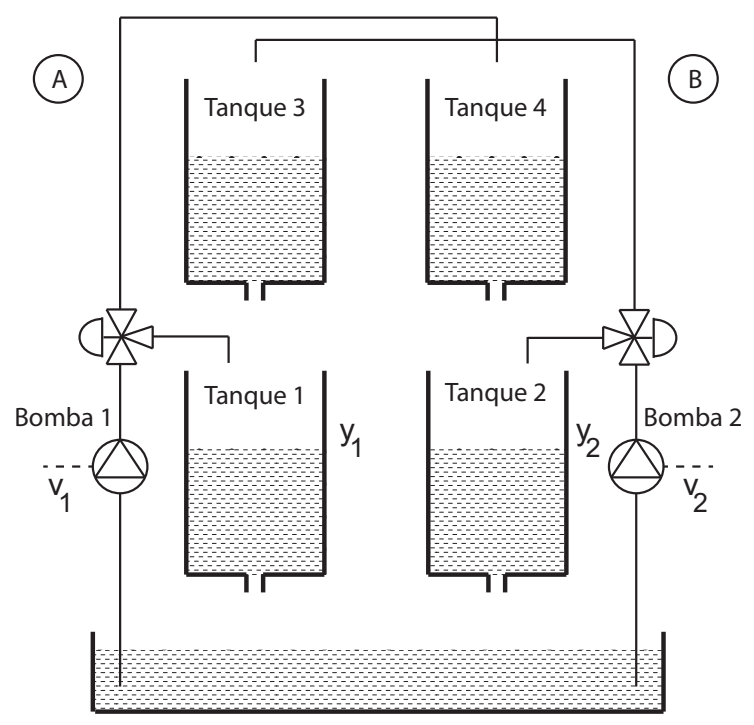

Figura 3. Processo de quatro tanques interconectados

No presente artigo, o objetivo, com este exemplo ilustrativo, é realizar o controle apenas do lado A do experimento original, considerando a perturbação do sistema sendo a influência exercida pelo sistema de bombeamento de fluido vindo do lado B da planta. É considerado ruído de medição tanto na simulação do sistema com os parâmetros originais, quanto para a simulação como o observador de perturbação. O controlador apresentado em Johansson (2000) é utilizado resultado de referências para análise das soluções encontradas.

A função de transferências que representa o modelo da planta $G$ é dada por:

$$
G(s)=\frac{2,6}{1+62 s}
$$

Já a função de transferência da perturbação, movida para entrada da planta, é descrita como:

$$
G_{d}(s)=\frac{1,5 / 2,6}{1+23 s}
$$

O algoritmo de otimização utilizado foi o DEMO, conforme já citado, sendo aplicado com objetivo de encontrar os valores mais adequados para o ganho proporcional, o tempo integral do controlador e para a constante do filtro do DOB. Para isto utilizou os seguintes parâmetros para simulação: tamanho da população $N=50$, realizando 100 iterações e com probabilidade de cruzamento igual a $90 \%$. O conjunto de soluções candidatas, ou seja, soluções que não são dominadas por nenhuma outra, são apresentadas na figura 4.

Cada ponto do gráfico representa uma possível solução eficiente para o problema, dentro do intervalo utilizado para otimização de cada uma das variáveis. O intervalo utilizado para o ganho proporcional foi $0,5<k_{p}<15$, para o tempo integral foi $0,01<1 / T_{i}<0,1$ e a constante do filtro de $0,1<T_{q}<20$. Essas faixas foram escolhidas por tentativa e erro para gerar uma curva de Pareto em uma região de interesse. 


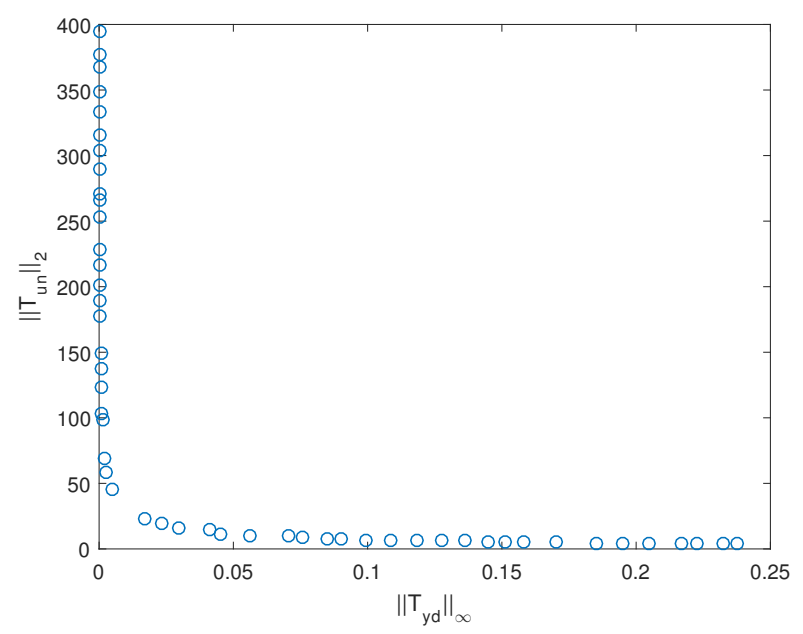

Figura 4. Curva de soluções candidatas de Pareto

As soluções obtidas e apresentadas no gráfico 4, localizadas na parte superior próximas ao eixo das ordenadas, em $\left\|T_{u n}\right\|_{2}$, são resultados com maior rejeição à perturbação, porém apresentam maior esforço de controle e menor atenuação do ruído. Já as soluções mais à direita em $\left\|T_{y d}\right\|_{\infty}$ requerem menor esforço de controle, apresentam maior atenuação do ruído, porém têm menor rejeição à perturbação. Assim, a escolha da melhor solução depende do compromisso escolhido entre esses objetivos.

A simulação foi realizada considerando um intervalo de tempo $0 \leq t \leq 400 s$. Primeiro foi feita uma variação no sinal de referência tipo degrau unitário. A perturbação, tipo pulso unitário, foi iniciada em $t=150$ e finalizada em $t=200$.

Em um primeiro momento foi escolhido os parâmetros do controlador de forma que o esforço de controle fosse aproximadamente igual ao esforço de controle utilizado por Johansson (2000), conforme figura 5. Com isso a saída observada na figura 6 , já apresenta uma maior rejeição à perturbação. O segundo teste realizado, em um outro cenário, que permite um esforço de controle maior, figura 7, é possível perceber que a perturbação é totalmente rejeitada na saída planta, conforme figura 8.

Uma breve comparação entre o observador de perturbação e as técnicas de controle clássico utilizadas para tratar sistemas com ruídos, distúrbios e erro de rastreamento se dá pelo fato que no controle clássico, o controlador, com um grau de liberdade, deve ser capaz de estabelecer um compromisso entre essas três respostas.

Quando é possível mensurar o distúrbio, pode-se ter um bloco que o trate separadamente, tendo assim, um controle com dois graus de liberdade. Porém, como nem sempre é possível medir os distúrbios presentes no sistema, com a aplicação do controle baseado em DOB, é possível realizar uma estimativa e tratar esses distúrbios em um bloco separado. A configuração de controle resultante, com dois graus de liberdade, dispensa a instalação de sensores, e requer estabelecer um compromisso apenas entre a resposta de rastreamento e ruído.

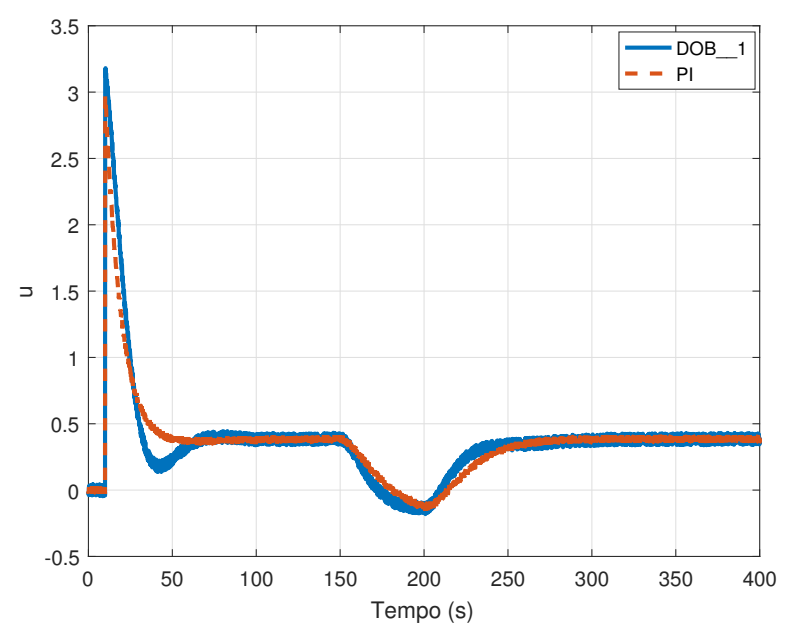

Figura 5. Esforço de controle para o DOB_1 proposto (sólida) e do controle proposto por Johansson (tracejado)

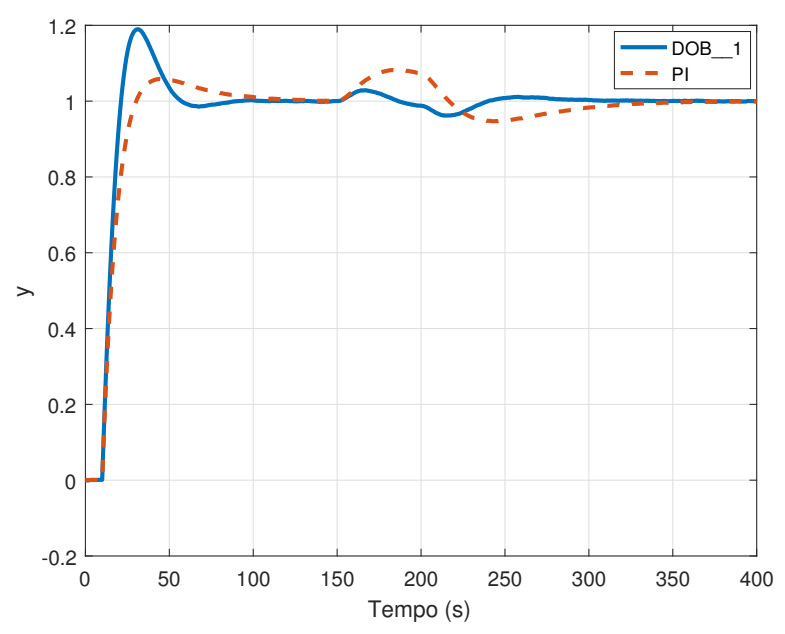

Figura 6. Resposta do Sistema para o DOB_1 proposto (sólida) e do controle proposto por Johansson (tracejado)

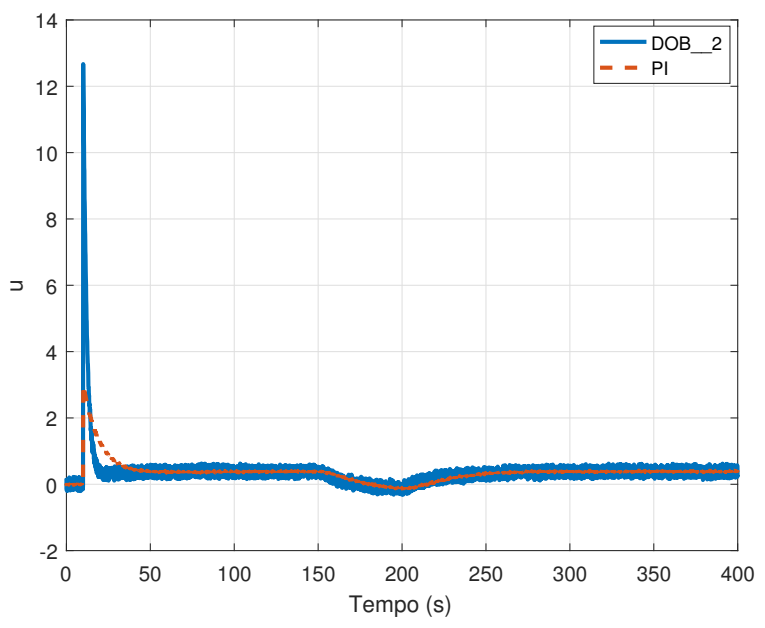

Figura 7. Esforço de controle para o DOB_1 proposto (sólida) e do controle proposto por Johansson (tracejado) 


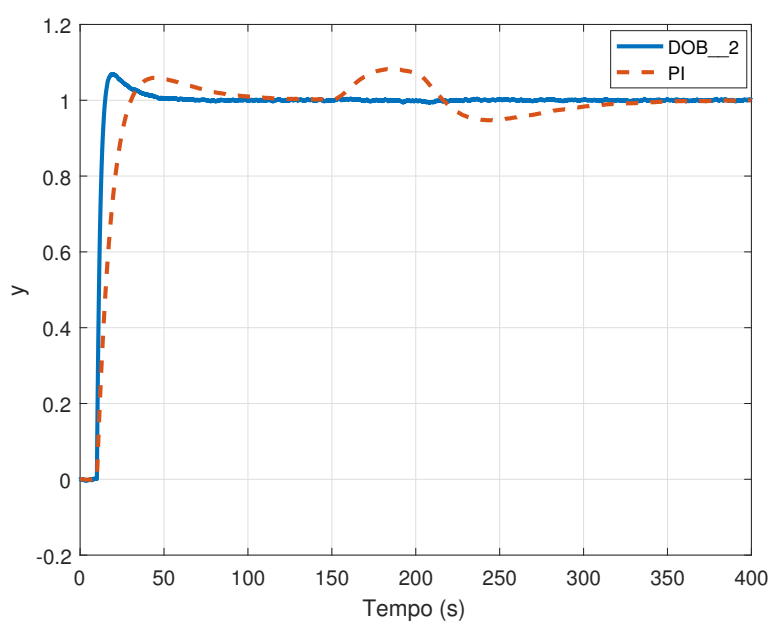

Figura 8. Resposta do Sistema para o DOB_1 proposto (sólida) e do controle proposto por Johansson (tracejado)

A avaliação entre os desempenhos obtidos em cada um dos casos foi realizada por meio do índice de desempenho ISE (do inglês, Integral of the Square of the Error):

$$
I S E=\int_{0}^{\infty} e^{2} d t
$$

Em que $e=r-y$ é o erro de rastreamento.

A tabela 1 apresenta, de forma sintetizada, o desempenho encontrado para cada uma das implementações realizadas. O controlador com observador de distúrbios DOB_1 corresponde a um projeto com ação de controle mais suave e maior atenuação de ruído, porém com maior erro devido a perturbação, como apresentado nas figuras 5 e 6 .

O controlador com observador de distúrbios DOB_2 corresponde a um projeto com ação de controle maior e maior influência de ruído, mas com menor erro devido a perturbação, como apresentado nas figuras 7 e 8, refletindo no menor valor de ISE dos três projetos listados. Como era esperado, quanto menor a constante de tempo de filtro, significando maior proximidade para $Q(s)=1$, melhor é a rejeição ao distúrbio. Como poder ser visto pelas figuras 6 a 8 , o método proposto fornece ao projetista um leque variado de soluções permitindo ao mesmo a escolha da solução que atende as suas prioridades.

Tabela 1. Desempenho do controlador PI e dos observadores de distúrbios.

\begin{tabular}{ccccc} 
& $I S E$ & $K_{p}$ & $1 / T_{i}$ & $T_{q}$ \\
\hline PI_original & 4,1610 & 3,0000 & 0.1000 & - \\
DOB_1 & 3,5067 & 3,1750 & 0,0995 & 18,3355 \\
DOB_2 & 0,9641 & 12,7343 & 0.0759 & 2.8976 \\
\hline
\end{tabular}

\section{CONCLUSÃO}

Neste trabalho, foi abordado uma estrutura de controle baseado em observador de distúrbio cujos parâmetros foram otimizados por meio do algoritmo DEMO. Com os resultados obtidos, por meio da minimização de objetivos conflitantes de controle, foi possível concluir que o observador de distúrbio consegue reduzir ou até mesmo eliminar as influências das perturbações, se for permitido um maior esforço de controle. A planta invertida no observador de distúrbio atua como um filtro passa-altas amplificando os ruídos de medição. O projeto do filtro passa-baixas do observador de distúrbio é fundamental para se obter o compromisso entre a rejeição de distúrbio, idealmente constante de tempo nula, e atenuação de ruídos de medição, constante de tempo elevada. A técnica de otimização permitiu determinar um conjunto de soluções candidatas que podem ser avaliadas para escolha da mais adequada para o problema específico.

\section{REFERÊNCIAS}

An, H., Liu, J., Wang, C., and Wu, L. (2016). Disturbance observer-based antiwindup control for air-breathing hypersonic vehicles. IEEE Transactions on Industrial Electronics, 63, 3038-3049.

Chen, W.H. (2004). Disturbance observer based control for nonlinear systems. IEEE/ASME Transactions on Mechatronics, 9, 706-710.

Chen, W.H., Yang, J., Guo, L., and Li, S. (2016). Disturbance observer based control and related methods - an overview. IEEE Transactions on Industrial Electronics, 63, 1083-1095.

Deb, K., Agarwal, A., and Meyarivan, T. (2002). A fast and elitist multiobjective genetic algorithm: Nsga-ii. IEEE Transactions on Evolutionary Computation, 6, $182-197$.

Guo, L. and Chen, W.H. (2005). Disturbance attenuation and rejection for systems with nonlinearity via dobc approach. IEEE Transactions on Evolucionary Computation, 15, 109-125.

Johansson, K. (2000). The quadruple-tank process: A multivariable laboratory process with an adjustable zero. IEEE Transactions on Control Systems Technology, 8, 456-465.

Mi, Y., Fu, Y., Li, D., Wang, C., Loh, P.C., and Wang, P. (2016). The sliding mode load frequency control for hybrid power system based on disturbance observer. International Journal of Electrical Power and Energy Systems, 74, 446-452.

Ohishi, K., Ohnishi, K., and Miyachi, K. (1983). Torque speed regulation of dc motor based on load torque estimation method. IEEE International Power Electronics Conference, 2, 1209-1218.

Price, K. and Storn, R. (1997). Differential evolution a simple evolution strategy for fast optimization. $D r$. Dobb's Journal, 22, 18-24.

Robič, T. and Filipič, B. (2005). Demo: Differential evolution for multiobjective optimization. Lecture Notes in Computer Science, 3410, 520-533.

Storn, R. and Price, K. (1997). Ddifferential evolution a simple and efficient adaptive scheme for global optimization over continuous spaces. ournal of Global Optimization, 11, 341-359.

Wang, L. and Su, J. (2015). Disturbance rejection control for non-minimum phase systems with optimal disturbance observer. ISA Transactions, 57, 1-9.

Zitzler, E., Laumanns, M., and Thiele, L. (2001). Spea2: Improving the strength pareto evolutionary algorithm for multiobjective optimization. Proc. Evolutionary Methods for Design Optimization and Control with Applications to Industrial Problems, 95-100. 